\title{
Incidência de tromboembolismo venoso em pacientes de um hospital especializado em Cardiopneumologia de alta complexidade
}

\author{
Venous thromboembolism incidence in patients in a hospital specialized in high complexity \\ Cardiopneumology
}

\begin{abstract}
Eduesley Santana Santos ${ }^{1}$, Luciana Soares Costa Santos ${ }^{2}$, Eldma de Jesus Fonseca Pereira ${ }^{3}$, Ingrid Isabel Matunaga ${ }^{3}$, Juliana de Lima Lopes ${ }^{4}$, Rita de Cassia Gengo e Silva ${ }^{4}$, Fatima Gil Ferreira ${ }^{4}$
\end{abstract}

\section{Resumo}

O tromboembolismo venoso (TEV) em seu espectro patológico inclui trombose venosa profunda, trombose associada ao uso de cateteres venosos e o tromboembolismo pulmonar (TEP), a forma mais grave. Pode ser assintomático e apresentar alto indice de mortalidade. Objetivo: avaliar a incidência de tromboembolismo venoso nos pacientes clínicos internados em um hospital especializado em Cardiopneumologia. Trata-se de um estudo transversal e retrospectivo, em que foram avaliadas informações dos pacientes a partir de um banco de dados existente derivadas de um coorte, realizado em um hospital escola especializado em Cardiopneumologia, de alta complexidade, na cidade de São Paulo, com pacientes adultos, internados em Unidades de Terapia Intensiva (UTI) e Unidades de Internação (UI). Foram selecionados 120 pacientes, divididos em dois grupos: pacientes que não desenvolveram tromboembolismo venoso (grupo não TEV) e pacientes que desenvolveram tromboembolismo venoso (grupo TEV). A incidência de TEV foi de 5,8\%; a maioria, em ambos os grupos, apresentava algum fator de risco para tromboembolismo venoso $(94,2 \%$ no grupo não TEV e

1. Professor da Universidade Federal de Sergipe (Campus Lagarto). Departamento de Enfermagem

2. Professora Instrutora da Faculdade de Ciências Médicas da Santa Casa de São Paulo. Curso de Graduação em Enfermagem. Professora da Escola de Enfermagem da Universidade de São Paulo (EEUSP). Professora do Centro Universitário das Faculdades Metropolitanas Unidas (FMU)

3. Aprimorandas do Curso de Cardiologia do InCOR - Instituto do Coração HC/FMUSP

4. Enfermeiras do Instituto do Coração do Hospital das Clínicas da Faculdade de Medicina da Universidade de São Paulo

Trabalho realizado: Instituto do Coração do Hospital das Clínicas da Faculdade de Medicina da Universidade de São Paulo - InCor-HC/FMUSP

Endereço para correspondência: Luciana Soares Costa Santos. Rua Dr. Cesário Mota Jr, 61 - $9^{\circ}$ andar - Vila Buarque-01221-020 - São Paulo-SP - Brasil.E-mail: luciana.santos@fcmsantacasasp.edu.br
100\% no grupo TEV), entretanto, o fator de risco de maior significância foi atribuído aos pacientes que necessitavam de terapia renal substitutiva (TRS) $(p=0,027)$. Não houve diferença entre os grupos com relação ao tipo de unidade onde os pacientes foram avaliados, na UI (60 vs. 71,4\%), na UTI clinica $(17,5$ vs. 14,3\%), na UCO $(15,8$ vs. $14,3 \%)$ e UTI cirúrgica (1,7 vs. 0) $p=0,964$. A mortalidade foi $4,1 \%$ contra $33,3 \%$, respectivamente nos grupos sem TEV e com TEV $(p=1,000)$. A mortalidade foi pequena nos dois grupos estudados, no período de internação. O estudo conclui que os pacientes submetidos à cirurgia cardiopulmonar apresentaram baixa incidência de TEV provavelmente devido ao emprego indireto da tromboprofilaxia, farmacológica e/ ou mecânica, todavia há necessidade de novos estudos para avaliar os critérios e métodos diagnósticos, bem como a profilaxia utilizada.

Descritores: Tromboembolia venosa, Incidência, Pacientes internados, Hospitalização, Fatores de Risco

\footnotetext{
Abstract

Venous thromboembolism (VTE) in its pathological spectrum includes deep venous thrombosis, and may also be associated with venous catheters and the most severe form, pulmonary thromboembolism. It can be asymptomatic and presents a high mortality rate. To identify the incidence of VTE in medical patients admitted to a hospital specialized in Cardiopneumology. This is a retrospective cross-sectional study in which we evaluated patient information from an existing database derived from a cohort, performed in a hospital specialized school in highly complex Cardiopneumology in São Paulo. The study included adult patients of adult intensive care units and wards.120 patients were selected for the study to analyze the patients were divided into two groups: VTE patients who did not (group not VTE) and patients who developed VTE (VTE group). The incidence of VTE was 5.8\%, most patients in both groups showed a risk factor for venous thromboembolism, and $94.2 \%$ in the group not TEV and $100 \%$ in the
} 
Santos ES, Santos LSC, Pereira EJF, Matunaga II, Lopes JL, Silva RCG, Ferreira FG. Incidência de tromboembolismo venoso em pacientes de um hospital especializado em Cardiopneumologia de alta complexidade. Arq Med Hosp Fac Cienc Med Santa Casa São Paulo. 2017;62(3):119-25.

TEV group, however, the factor of greater significance risk was assigned to patients in need of TRS $(p=0.027)$. There was no difference between groups in terms of the type of unit where patients were evaluated in the general inpatient unit (60 vs. $71.4 \%$ ) in clinical intensive care unit (17.5 vs. $14.3 \%)$, the coronary unit (15.8 vs. $14.3 \%$ ) and surgical intensive care unit (1.7 vs. 0) $p=0.964$. Mortality was 4.1 vs. 33.3\%, respectively in the groups with and without VTE $(p=1.000)$. Mortality was little in both groups that were studied during the hospitalization period. The study concludes that patients that were submitted to cardiopulmonary surgery presented low incidence of VTE, probably because of the indirect use of thromboprophylaxis, pharmacological and/or mechanical, although new studies need to be conducted to evaluate the criterion and methods diagnosed, as well as the prophylaxis used. VTE was manifested in $5.9 \%$ of patients analyzed, unlike the results found in the literature, since $95 \%$ of the participants have to work a risk factor. Although several patients have remained institutionalized for long periods mortality was low in both groups, we understand that this phenomenon was due to the indirect use of thromboprophylaxis, since most of the clients served at service, according to the medical diagnosis made during the admission, whether pharmacological, or mechanical. The study concludes that patients undergoing cardiopulmonary surgery had a low incidence of VTE in the patient, however, there is a need for new studies to evaluate the diagnostic criteria and methods, as well as the prophylaxis used.

Keywords: Venous thromboembolism, Incidence, Inpatients, Hospitalization, Risk factors

\section{Introdução}

Em 1856 Virchow $^{(1)}$ já descrevia que a estase venosa, a injúria da parede vascular e a coagulabilidade sanguínea aumentada favoreceriam o tromboembolismo venoso (TEV), surgindo então, a tríade de Virchow ${ }^{(2-6)}$.

Sabe-se que esta alteração é composta por doenças como a trombose venosa profunda (TVP), as tromboses relacionadas a cateteres venosos centrais e o tromboembolismo pulmonar (TEP). Suas principais complicações são a síndrome pós trombótica e a hipertensão pulmonar ${ }^{(3,7)}$.

Na fase aguda, a TVP é grave devido ao risco de embolias pulmonares fatais, frequentemente encontradas em 50\% dos casos com a patologia, contudo, o deslocamento de algum trombo alojado nas artérias pulmonares pode comprometer a função pulmonar e cardíaca diretamente ${ }^{(2,5,6)}$.

O óbito comumente é o agravo de maior relevância: cerca de 9 a 21\% dos corpos necropsiados apresentaram TEP, segundo estudo realizado ${ }^{(8)}$. Nos Estados Unidos aproximadamente 200 mil pessoas morrem anualmente em decorrência da $\mathrm{TEV}^{(2,9,10)}$. No
Brasil, os estudos sobre o TEV são escassos, contudo, estima-se que haja uma incidência de 0,6 casos por mil habitantes ao ano ${ }^{(11)}$.

Sabe-se que no ano de 2013, houve 47.294 internações ocasionadas por TEV, 2.315 óbitos, sendo a taxa de mortalidade para TEP de 21,66 por 100.000 , ocupando a oitava maior taxa, atrás apenas de algumas neoplasias, sepse e hemorragia intracraniana ${ }^{(12)}$. Sua incidência é cem vezes maior entre os indivíduos hospitalizados, principalmente em leitos de terapia intensiva (TI), quando comparados a indivíduos não hospitalizados $^{(3,7,13-14)}$.

A profilaxia para TEV, segundo a Diretriz de Tromboembolismo Venoso (2009), está recomendada para pacientes clínicos agudos, hospitalizados, com idade maior que 40 anos, com mobilidade reduzida e com pelo menos um fator de risco adicional para TEV. Pacientes jovens ( $<40$ anos), mas com importantes fatores de risco podem se beneficiar da profilaxia. A profilaxia com anticoagulantes deve ser utilizada por 6 até 14 dias, mesmo que o paciente comece a mobilização precoce ou receba alta. Novos anticoagulantes orais, atualmente em fase de estudo são promissores e podem tornar-se uma opção na profilaxia do TEV em pacientes clínicos. Os riscos de sangramentos devem sempre ser avaliados junto com o quadro clinico, e a indicação de profilaxia para TEV deve ser indicada sempre para os pacientes e não apenas para as doenças ${ }^{(7)}$.

Diante deste panorama alarmante, a Associação Médica Brasileira (AMB) e o Conselho Federal de Medicina (CFM) construíram um instrumento para avaliação de pacientes clínicos com médio e alto risco para TEV ${ }^{(7,14-16)}$. O Projeto Diretrizes $(2009)^{(7)}$ recomenda a instituição precoce da tromboprofilaxia em pacientes clínicos, a fim de reduzir de modo significativo o risco para TEV.

Incluem-se entre os fatores de risco comuns a esta população, atendida em um hospital terciário especializado em Cardiopneumologia, a idade, internação em Unidade de Terapia Intensiva, uso de cateteres venosos centrais, para hemodiálise e de artéria pulmonar (Swan-Ganz), insuficiência cardíaca congestiva classes III-IV, infarto agudo do miocárdio (IAM), acidente vascular encefálico isquêmico e ou hemorrágico (AVEI / $\mathrm{AVEH})^{(7)}$.

Alguns autores questionam a subutilização ou o uso incorreto da profilaxia para tromboembolismo $(3,10,13)$. Considerando os achados, pode-se inferir que as causas do TEV podem ser evitadas por meio de medidas farmacológicas e ou mecânicas e ainda reforçam o papel dos profissionais de saúde, como responsáveis por desenvolver estratégias que visem à redução dos casos de TEV em pacientes hospitalizados, visto que, o evento cursa com maior gravidade em pacientes clínicos quando comparados aos pacientes cirúrgicos ${ }^{(9,14,16)}$. 
Santos ES, Santos LSC, Pereira EJF, Matunaga II, Lopes JL, Silva RCG, Ferreira FG. Incidência de tromboembolismo venoso em pacientes de um hospital especializado em Cardiopneumologia de alta complexidade. Arq Med Hosp Fac Cienc Med Santa Casa São Paulo. 2017;62(3):119-25.

O estudo tem como objetivo, identificar a incidência de tromboembolismo venosos em pacientes clínicos internados em um hospital especializado em Cardiopmeumologia.

\section{Material e Método}

Trata-se de um estudo retrospectivo, observacional de corte transversal, realizado no período de janeiro de 2012 a maio de 2013, no Instituto do Coração (InCor) do Hospital das Clínicas da Faculdade de Medicina da Universidade de São Paulo (HC-FMUSP), hospital especializado em Cardiopneumologia. As informações dos pacientes foram obtidas a partir de um banco de dados derivados de um estudo transversal realizado previamente na instituição.

A amostra foi constituída por 120 pacientes das Unidades de Terapia Intensiva (UTI) Adulto e Unidades de Internação (UI), dentro do período de estudo, obtido por meio de amostragem por conveniência, com problemas cardiológicos e/ou pneumológicos.

Os critérios para inclusão no estudo foram pacientes internados para tratamento clínico, nas Unidades de Terapia Intensiva e Unidades de Internação, maiores de 18 anos com problemas cardiológicos e/ ou pneumológicos. Já os critérios para exclusão compreendiam pacientes internados para tratamento de outras patologias que não cardíacas e/ou pulmonares e TEV como motivo para internação no momento da coleta de dados.

O projeto de pesquisa foi submetido à Comissão Cientifica do Instituto do Coração do Hospital das Clínicas da Faculdade de Medicina da Universidade de São Paulo (InCor) e à Comissão de Ética para Análise de Projetos de Pesquisa (CAPPesq) do (HCFMUSP) e aprovado sob o número 3508/10/097.

Os pacientes foram divididos, conforme a clínica de internação e nomeados como pacientes que não desenvolveram TEV (grupo não TEV) e pacientes que desenvolveram TEV (grupo TEV).

Utilizou-se a técnica de análise de banco de dados e um questionário, conforme roteiro previamente elaborado, aplicado o algoritmo sugerido pela Associação Médica Brasileira (AMB) e o Conselho Federal de Medicina $(\mathrm{CFM})^{(7)}$ contendo variáveis para caracterização clínico-demográficas (idade, gênero, raça, índice de massa corporal - IMC, comorbidades como insuficiência cardíaca, hipertensão arterial sistêmica - HAS, diabetes mellitus - DM, dislipidemia - DLP, tabagismo atual ou prévio, fibrilação atrial - FA, hipotireoidismo, infarto agudo do miocárdio prévio - IAM, cirurgia cardíaca prévia, creatinina $>1.5 \mathrm{mg} / \mathrm{dL}$, fração de ejeção do ventrículo esquerdo - FEVE - entre 20 a $40 \%$, uso de betabloqueadores, inibidores da enzima conversora da angiotensina - IECA, bloqueador do receptor da angiotensina - BRA, razão normalizada internacional - INR alterado).

Além dos dados citados, foram compilados dados pertinentes ao uso de tromboprofilaxia farmacológica (heparina não fracionada ou heparina de baixo peso molecular) segundo as Diretrizes da AMB, uso de tromboprofilaxia mecânica (meias de compressão graduada), presença de fatores de risco para TEV nos pacientes avaliados, tempo de internação hospitalar, tempo de internação em UTI ou UI e mortalidade, de acordo com o fluxograma preconizado pela Diretriz da $\mathrm{AMB}^{(7)}$.

O desfecho primário foi a presença de TEV. Os desfechos secundários foram tempo de internação em UTI Clínica ou Cirúrgica ou UCO, tempo de internação em enfermaria, tempo de internação hospitalar e mortalidade. Os dados foram expressos em número absoluto, porcentagem, média, desvio padrão da média, mediana, valores mínimos e máximos.

Para verificar a normalidade das variáveis contínuas foi utilizado o teste de Kolmogorov-Smirnov. A diferença entre os grupos foi avaliada utilizando-se os testes T Bicaudal, U de Mann-Whitney, Qui-quadrado e exato de Fisher, conforme apropriado. Um valor de desvio padrão $(p)<0,05$ foi considerado significante. O programa estatístico SPSS versão 20.0 foi utilizado para a análise dos dados.

\section{Resultados e Discussão}

O TEV é uma patologia de importante prevalência em pacientes clínicos hospitalizados, de caráter multifatorial e silenciosa, ocasionando sequelas no longo prazo. Por vezes, esse evento embólico pode ser fatal quando a TVP evolui para TEP $(2,3,6,15)$.

Os pacientes clínicos são os mais acometidos por esses fenômenos, pois a longa permanência em uma instituição hospitalar os deixa vulneráveis. Sendo assim, a presença de pelo menos um dos componentes da tríade de Virchow, em associação a algum fator de risco, propiciam o desenvolvimento de TEV conforme descrito na literatura ${ }^{(3,5,7,14,16-17)}$. Deste modo, os estudos demonstram que a cada década os índices de TEV aumentam, corroborando os dados encontrados ${ }^{(2,3,6,10)}$.

Estudos realizados ${ }^{(10,13-16)}$ corroboraram uma alta incidência de tromboembolismo venoso, havendo consenso de que são 1,2 casos/ mil habitantes/ ano nos EUA, 1,6 casos/ mil habitantes / ano na Suécia e 0,6 casos / mil habitantes/ ano no Brasil.

Diogo-Filho et al., $2009^{(11)}$, afirmam que a real incidência da doença permanece desconhecida, devido à sua história natural e por evoluir frequentemente de maneira insidiosa ou com sinais e sintomas comuns a outras doenças, o que contribui para subnotificação, acrescido ao fato de que a maioria dos estudos 
Santos ES, Santos LSC, Pereira EJF, Matunaga II, Lopes JL, Silva RCG, Ferreira FG. Incidência de tromboembolismo venoso em pacientes de um hospital especializado em Cardiopneumologia de alta complexidade. Arq Med Hosp Fac Cienc Med Santa Casa São Paulo. 2017;62(3):119-25.

epidemiológicos se baseia no diagnóstico clínico de sensibilidade inferior a 50,0\%.

Argenta $(2007)^{(17)}$ em seu estudoaponta que a incidência de tromboembolismo pulmonar (TEP) é maior em pacientes que apresentam doenças cardíacas, em pacientes sem profilaxia para TEV e nos submetidos à cirurgia de revascularização miocárdica, com cerca de $4 \%$ de TEP. Rassam et al, $2009^{(18)}$ ressaltam que a taxa de mortalidade para embolia pulmonar é alta e aponta em seu estudo que cerca de 70 a $80 \%$ das embolias pulmonares diagnosticadas na necropsia não tinham suspeita clínica prévia, como o caso encontrado nesta pesquisa. Entretanto, o TEP pode ser um dos fatores desencadeantes (5T) de uma parada cardiorrespiratória (PCR), principalmente, em casos de assistolia. Na Tabela 1, apresenta-se as características clínicas e demográficas dos pacientes avaliados no estudo.

Dos 51 pacientes analisados, 94,1\% (48) deles tinha pelo menos um fator de risco para TEV, $27,4 \%$ eram portadores de insuficiência cardíaca (IC), 72,5\% de hipertensão arterial sistêmica (HAS), 41,2\% diabetes mellitus (DM), 52,9\% dislipidemia (DLP), 9,8\%, eram tabagistas, a fibrilação atrial (FA) esteve presente em 9,8\% dos casos (sendo esta a comorbidade que acometeu menor número de indivíduos), 25,5\% dos avaliados tinham diagnóstico de IAM prévio, $29,4 \%$ foram submetidos à cirurgia cardíaca prévia, $29,4 \%$ apresentavam creatinina sérica maior que 1,5 $\mathrm{mg} / \mathrm{dL}$, o índice internacional normalizado (INR) à admissão apresentou média de 1,22 $\pm 0,18$, a fração de ejeção do ventrículo esquerdo (FEVE) foi de 61\%, com variação mínima de 20 e máxima de $76 \%$. Das cirurgias realizadas, 21 dos pacientes $(41,2 \%)$ foram submetidos à revascularização miocárdica (RM), 12 $(23,5 \%)$ a cirurgias da aorta, $10(19,6 \%)$ realizaram algum tipo de cirurgia valvar, sendo o tempo de circulação extracorpórea (CEC) duração média de $103,3 \pm 36,6$ minutos

As Tabelas 1 e 2 apontam para a presença de fatores de risco em quase todos os pacientes, conforme esperado. A literatura descreve que quase todos os pacientes hospitalizados apresentam pelo menos um fator de risco para desenvolver TEV e, aproximadamente, $40 \%$ têm três ou mais. Os pacientes cirúrgicos possuem fatores de risco aumentados para desenvolver a doença, inerentes ao próprio procedimento cirúrgico e ao aumento da frequência de cirurgias combinadas, indo ao encontro dos resultados: oito pacientes foram submetidos à cirurgia de revascularização miocárdica associada à troca de valva, necessitando assim, de internação em UTI, o que agregou mais um fator de risco ${ }^{(19)}$. Já na Tabela 2, estão apresentados os fatores de risco para o tromboembolismo venoso identificados no estudo.

Na Tabela 3, são apresentados os dados relacio-

\begin{tabular}{lc}
\multicolumn{2}{c}{ Tabela 1 } \\
\multicolumn{2}{c}{ Características clínicas e demográficas dos pacientes } \\
\multicolumn{2}{c}{ avaliados no estudo - InCor HC-FMUSP, 2015.} \\
Resultado \\
Características Clínicas
\end{tabular}

Legenda: IMC: índice de massa corpórea; TEV: tromboembolismo venoso; HAS: hipertensão arterial sistêmica; DM: diabetes mellitus; DLP: dislipidemia; IAM: infarto agudo do miocárdio; INR: índice internacional normalizado; FEVE: fração de ejeção do ventrículo esquerdo; EuroSCORE: Europa System for Cardiac Operative; IECA: inibidor da enzima conversora da angiotensina; BRA: bloqueador do receptor da angiotensina; CEC: circulação extracorpórea UTI: Unidade de Terapia Intensiva.

nados à análise multivariada do grupo com TEV e do grupo sem TEV.

Na análise da Tabela 3 verifica-se que os resultados encontrados contradizem os dados da literatura, uma vez que no InCor houve baixa frequência de 


\section{Tabela 2}

Fatores de Risco para TEV* - InCor HC-FMUSP, 2015.

\begin{tabular}{|c|c|}
\hline Variável & $\begin{array}{c}\text { Resultado } \\
(n=51)\end{array}$ \\
\hline AVC & $3(5,9 \%)$ \\
\hline Neoplasia & $2(3,9 \%)$ \\
\hline Cateter Central & $27(52,9 \%)$ \\
\hline IAM & $14(27,5 \%)$ \\
\hline ICC classe III ou IV & $13(25,5 \%)$ \\
\hline Idade $>55$ anos & $28(54,9 \%)$ \\
\hline Infecção & $26(51 \%)$ \\
\hline Insuficiência Arterial Periférica & $1(1,7 \%)$ \\
\hline Internação em UTI & $45(88,2 \%)$ \\
\hline Obesidade (IMC > $\left.30 \mathrm{~kg} / \mathrm{m}^{2}\right)$ & $13(25,5 \%)$ \\
\hline Varizes & $4(7,8 \%)$ \\
\hline
\end{tabular}

AVC: acidente vascular cerebral; IAM: infarto agudo do miocárdico; ICC: insuficiência cardíaca congestiva; UTI: Unidade de Terapia Intensiva; IMC: Índice massa corpórea. *Fonte: Geerts et al 2008(20) , Associação Médica Brasileira, Conselho Federal de Medicina, 2005(21) , Rocha et al, 2006 (22), NICE, 2015(23)
TEV diagnosticado, sendo que apenas três pacientes dos 51 desenvolveram TVP, baseado em critérios clínicos e confirmados com o uso de Ultrassonografia Dopller e Tomografia. Contudo, houve um caso de TEV diagnosticado apenas na necropsia, em que o paciente apresentou TEP que, conforme o laudo foi desenvolvido no período correspondente à internação, porém, sem suspeita clínica, diagnóstico ou profilaxia, evoluindo para morte súbita após PCR.

Por se tratar de uma população de cardiopatas, a insuficiência cardíaca não foi considerada como fator de risco; contudo, os demais fatores encontrados com maior frequência, são semelhantes aos apontados pela literatura ${ }^{(24)}$.

Ao compararmos os dados dos grupos com e sem TEV, os desfechos apresentados relacionavam a taxa de mortalidade, tempo de internação hospitalar, UTI e enfermaria conforme a Tabela 4.

Os resultados apontam para maior tempo de hospitalização e internação em UTI, conforme Tabela 4. para pacientes que desenvolveram TEV. Esse achado é justificado pelo contexto da internação em UTI, que agrega outros fatores de risco, como imobilidade do paciente ao leito, sedação, ventilação mecânica, uso

Tabela 3

Análise univariada dos pacientes que desenvolveram e os que não desenvolveram TEV - InCor HC-FMUSP, 2015.

\begin{tabular}{|c|c|c|c|}
\hline Variável & $\begin{array}{c}\text { Sem TEV } \\
(n=48)\end{array}$ & $\begin{array}{c}\text { Com TEV } \\
(n=3)\end{array}$ & $p$ (valor) \\
\hline Gênero Feminino & $24(50 \%)$ & $2(66,7 \%)$ & 1,000 \\
\hline Idade (anos) & $56 \pm 15$ & $59 \pm 4$ & 0,725 \\
\hline $\operatorname{IMC}\left(\mathrm{kg} / \mathrm{m}^{2}\right)$ & $25,5 \pm 3,7$ & $22,2 \pm 2,8$ & 0,215 \\
\hline EuroSCORE & $6,4 \pm 2,5$ & $5,3 \pm 1,1$ & 0,477 \\
\hline FEVE & $55,8 \pm 14,2$ & $54,3 \pm 12$ & 0,170 \\
\hline Insuficiência Cardíaca & $13(27,1 \%)$ & $1(33,3 \%)$ & 0,818 \\
\hline Hipertensão & $35(68,5 \%)$ & $2(66 \%)$ & 1,000 \\
\hline Dislipidemia & $24(51,1 \%)$ & $2(66,7 \%)$ & 1,000 \\
\hline Tabagismo atual & $4(8,3 \%)$ & $1(33,3 \%)$ & 0,271 \\
\hline Tabagismo prévio & $9(18,8 \%)$ & $1(33,3 \%)$ & 0,488 \\
\hline Creatinina $(>1,5 \mathrm{mg} / \mathrm{dL})$ & $14(29,2 \%)$ & $1(33,3 \%)$ & 1,000 \\
\hline $\mathrm{DM}$ & $19(39,6 \%)$ & $2(66,7 \%)$ & 0,561 \\
\hline Fatores de Risco & $45(93,8 \%)$ & $3(100 \%)$ & 1,000 \\
\hline$I N R$ & $1,22 \pm 0,18$ & $1,16 \pm 0,11$ & 0,621 \\
\hline Creatinina à Admissão & $1,5 \pm 1,0$ & $1,2 \pm 0,4$ & 0,620 \\
\hline \multicolumn{4}{|l|}{ Intra-operatório } \\
\hline Tempo de CEC (min) & $100(31-200)$ & $116(75-200)$ & 0,185 \\
\hline Balanço Hídrico $(m L)$ & $2490(250-3950)$ & $3450(1100-4300)$ & 0,371 \\
\hline Diurese $(m L)$ & $1200(0-3600)$ & $1400(1400-3500)$ & 0,038 \\
\hline
\end{tabular}

Legenda: IMC: índice de massa corpórea; FEVE: fração de ejeção ventrículo esquerdo; DM: diabetes mellitus; INR: Índice internacional normalizado; CEC: circulação extracorpórea. 


\begin{tabular}{lccc}
\hline \multicolumn{4}{c|}{ Tabela 4} \\
Comparação entre o desfecho de pacientes do grupo TEV e do grupo não TEV - InCor HC-FMUSP, 2015. \\
\hline Desfecho & $\operatorname{Sem}$ TEV $(n=48)$ & Com TEV $(n=3)$ & $p$ (valor) \\
Mortalidade (\%) & $2(4,1)$ & $1(33,3)$ & 1,000 \\
Tempo de Internação Hospitalar (dias) & $28(4-131)$ & $42(33-42)$ & 0,934 \\
Tempo de Internação em UTI (dias) & $6(1-68)$ & $17(14-27)$ & 0,397 \\
Tempo de Internação em Enfermaria (dias) & $18(2-115)$ & $19(15-25)$ & 0,682 \\
\hline
\end{tabular}

Legenda: UTI: unidade de terapia intensiva

de cateteres venoso central, Swan-Ganz e de diálise, frequentemente implantados em pacientes críticos/ instáveis, que necessitam de monitorização invasiva e drogas vasoativas.

Na comparação do desfecho entre os dois grupos, a incidência de mortalidade foi maior entre os pacientes que apresentaram TEV, encontrou-se um paciente (33,3\%) e no grupo sem TEV, dois $(4,1 \%), p=1,000$. Constatou-se que o tempo médio de internação hospitalar foi de 28 dias (variando entre 4 a 131 dias) nos pacientes sem TEV e 42 dias (variando entre 33 a 42) dias nos pacientes com TEV $(p=0,934)$, sendo que os pacientes que não apresentaram TEV tiveram tempo médio de 6 dias (com variação entre 1 e 68 dias) internados em Unidades de Terapia Intensiva e os com TEV de 17 dias (14 - 27) ( $\mathrm{p}=0,397)$, e o tempo de internação em enfermarias pelos pacientes do grupo que não apresentou TEV foi de 18 dias (2 - 115) sem diferença significativa em comparação com o grupo que desenvolveu $\mathrm{TEV}$, que permaneceu internado em média 19 dias $(15-25)(p=0,682)$. Os dados estatísticos demonstram que nenhuma das variáveis apresentou $\mathrm{p}$ relevante.

Quanto à mortalidade por TEV a literatura cita que esta doença é a maior e mais frequente causa não reconhecida de morbimortalidade em pacientes hospitalizados $^{(25-26)}$. Os achados de Diogo-Filho et al, 2009(11), vão ao encontro dos resultados deste estudo, em relação à não detecção clínica de sinais e sintomas de TEV, uma vez que pode haver pacientes com TEV sem diagnóstico, sendo que o diagnóstico definitivo se fará apenas se o paciente evoluir com TEP ou durante necropsia. Em casos de PCR, o óbito poderia ser evitado diante da suspeita clínica e tratamento empírico e confirmado por ecocardiograma transtorácico. Diante da confirmação diagnóstica, deve-se iniciar a administração de trombolítico mas, ainda assim, sem garantia de evitar hipertensão pulmonar, insuficiência valvar ou mesmo embolia pulmonar como complicações. Contudo, a profilaxia adequada da causa do TEV exalta o TEP como a principal causa prevenível de óbito intra-hospitalar ${ }^{(4)}$.

\section{Conclusão}

Diante dos achados deste estudo, concluiu-se que, apesar dos pacientes sem TEV apresentarem EuroSCO-
RE com alto risco de mortalidade pós cirurgia cardíaca, a mortalidade foi mais frequente em pacientes que desenvolveram TEV com médio risco.

\section{Referências}

1. Virchow RLK. La pathologie cellulaire based sur l'étude pathologique des tissus. $3^{\mathrm{a}}$ ed. Paris: Baillière; 1868. 456p.

2. Busato CR, Gomes RZ, Costa DMM, Zubiolo TFM. Avaliação de tromboprofilaxia em hospital geral de médio porte. J Vasc Bras. 2014; 13(1):5-11.

3. Baer C, Bobato C, Carvalho M, Frandoloso G, Gabardo LC, Rauber E, et al. Avaliação da profilaxia medicamentosa do tromboembolismo venoso. Rev Soc Bras Clin Med. 2012; 10(5):372-6.

4. Smeltzer SC, Bare BG. Histórico e tratamento de pacientes com distúrbios vasculares e problemas da circulação periférica. In: Smeltzer SC, Abre BG. Brunner \& Suddarth tratado de enfermagem medico-cirúrgica. 9a. ed. Rio de Janeiro: Guanabara Koogan; 2002. v.2, p. 680.

5. Machado NLB, Leite TL, Pitta GBB. Frequência da profilaxia mecânica para trombose venosa profunda em pacientes internados em uma unidade de emergência de Maceió. J Vasc Bras. 2008; 7(4):333-40.

6. Rodrigues MM. Epidemiologia da tromboembolia venosa. Pneumol Paulista. 2012; 26(4):7-9.

7. Academia Brasileira de Neurologia, Associação de Medicina Intensiva Brasileira, Federação Brasileira das Associações de Ginecologia e Obstetrícia, Sociedade Brasileira de Angiologia e de Cirurgia Vascular, Sociedade Brasileira de Cancerologia, Sociedade Brasileira de Cardiologia, et al. Tromboembolismo venoso: profilaxia em pacientes clínicos - parte I. Rev Assoc Med Bras. 2009; 55(2):102-5.

8. Andrade EO, Bindá FA, Silva AMM, Costa TDA, Fernandes $\mathrm{MC}$, Fernandes MC. Fatores de risco e profilaxia para tromboembolismo venoso em hospitais da cidade de Manaus. J Bras Pneumol. 2009; 35(2):114-21.

9. Haga CS, Mancio CM, Pioner MC, Alves FAL, Lira AR, Silva JS, et al. Implantação do serviço do farmacêutico clínico vertical na profilaxia do tromboembolismo venoso em pacientes clínicos hospitalizados. Einstein (São Paulo). 2014; 12(1):27-30.

10. Pitta GBB, Gomes RR. A frequência da utilização de profilaxia para trombose venosa profunda em pacientes clínicos hospitalizados. J Vasc Bras. 2010; 9(4):220-8.

11. Diogo-Filho A, Maia CP, Diogo DM, Fedrigo LSP, Diogo PM, Vasconcelos PM. Estudo de vigilância epidemiológica da profilaxia do tromboembolismo venoso em especialidades cirúrgicas de um hospital universitário de nível terciário. Arq Gastroenterol. 2009; 46(1):9-14.

12. Brasil. Ministério da Saúde. DATASUS - Departamento de Informática do SUS. [online]. Informações de Saúde, Sistema de Informações sobre Mortalidade. Disponível em: http:/ / www2. datasus.gov.br/DATASUS/index.php?area=0203 [22 jan 2015] 
13. Rocha ATCC, Paiva EF, Araújo DM, Cardoso DN, Pereira ACH, Lopes AA, et al. Impacto de um programa para profilaxia de tromboembolismo venoso em pacientes clínicos em quatro hospitais de Salvador. Rev Assoc Med Bras. 2010;56(2):197-203.

14. Tapson VF, Decousus H, Pini M, Chong BH, Froehlich JB, Monreal $\mathrm{M}$, et al. Venous thromboembolism prophylaxis in acutely ill hospitalized medical patients: findings from the International Medical Prevention Registry on Venous Thromboembolism. Chest. 2007; 132(3):936-45.

15. Kerbauy MN, Moraes FY, Kerbauy KN, Conterno LO, El-Fakhouri S. Tromboprofilaxia venosa em pacientes clínicos: análise de sua aplicação. Rev Assoc Med Bras. 2013; 59(3):258-64.

16. Cohen AT, Tapson VF, Bergmann JF, Goldhaber SZ, Kakkar AK, Deslandes B, et al. Venous thromboembolism risk and prophylaxis in the acute hospital care setting (ENDORSE study): a multinational cross-sectional study. Lancet. 2008;371(9610):38794.

17. Argenta C. Análise de custo-minimização do uso de heparina não-fracionada e enoxoparina em uma coorte de pacientes em tratamento de tromboembolismo venoso. Dissertação (Mestrado). Porto Alegre: Faculdade de Medicina - Universidade Federal do Rio Grande do Sul; 2007.

18. Rassam E, Pinheiro TC, Stefan LFB, Modena SF. Complicações tromboembólicas no paciente cirúrgico e sua profilaxia. ABCD Arq Bras Cir Dig. 2009; 22(1):41-4.

19. Paiva RA, Pitanguy I, Amorim NFG, Berger R, Shdick HA, Holanda TA. Tromboembolismo venoso em cirurgia plástica: protocolo de prevenção na Clínica Ivo Pitanguy. Rev Bras Cir Plást. 2010; 25(4):583-8.

20. Geerts WH, Bergqvist D, Pineo GF, Heit JA, Samama CM, Lassen MR, et al. Prevention of venous thromboembolism: American College of Chest Physicians Evidence-Based Clinical Practice Guidelines (8th Edition). Chest. 2008 Jun;133(6 Suppl):381S453S.
21. Associação Médica Brasileira, Conselho Federal de Medicina. Tromboembolismo venoso: profilaxia em pacientes clínicos. [online]. Elaboração final: 31 de março de 2005. Disponível em: http://diretrizes.amb.org.br/diretrizes [ 20 nov 2015].

22. Rocha AT, de Vasconcellos AG, da Luz Neto ER, Araújo DM, Alves ES, Lopes AA. Risk of venous thromboembolism and efficacy of thromboprophylaxis in hospitalized obese medical patients and in obese patients undergoing bariatric surgery. Obes Surg. 2006; 16(12):1645-55.

23. NICE - National Institute for Health and Care Executive. Venous thromboembolism: reducing the risk for patients in hospital. [online]. Clinical guideline [CG92] Published date: January 2010 Last updated: June 2015. Available from: https://www. nice.org.uk/guidance/cg92 [2015 Nov 2]

24. Kayali F, Najjar R, Aswad F, Matta F, Stein PD. Venous thromboembolism in patients hospitalized with nephrotic syndrome. Am J Med. 2008; 121(3):226-30.

25. Lanfranchi JRA, Sardinha WE, Silvestre JMS, Palma O, Morais Filho DMF, Silva MAF, Embolia pulmonar em necropsias no Hospital Universitário Regional do Norte do Paraná. Incidência em 10 anos e correlação clínico-anátomo-patológico. Cir Vasc Angiol. 1995; 11(Supl 1):12.

26. Academia Brasileira de Neurologia, Associação de Medicina Intensiva Brasileira, Federação Brasileira das Associações de Ginecologia e Obstetrícia, Sociedade Brasileira de Angiologia e de Cirurgia Vascular, Sociedade Brasileira de Cancerologia, Sociedade Brasileira de Cardiologia, et al. Tromboembolismo Venoso: Profilaxia em Pacientes Clínicos - Parte III. Rev Assoc Med Bras. 2009; 55(4):363-81.

Trabalho recebido: $15 / 08 / 2016$

Trabalho aprovado: 21/10/2017 